\title{
Displacement of the Hip Center of Rotation After Arthroplasty of Crowe III and IV Dysplasia: A Radiological and Biomechanical Study
}

\section{Mansour Abolghasemian}

Tehran University of Medical Sciences

\section{Saeid Samiezadeh}

Ryerson University

\section{Davood Jafari}

Tehran University of Medical Sciences

\section{Habiba Bougherara}

Ryerson University

\section{Allan E. Gross}

Mount Sinai Hospital

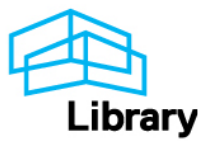




\title{
Displacement of the Hip Center of Rotation After Arthroplasty of Crowe III and IV Dysplasia: A Radiological and Biomechanical Study
}

\author{
Mansour Abolghasemian MD ${ }^{\text {a,b }}$, Saeid Samiezadeh PhD Candidate ${ }^{c}$, Davood Jafari MD ${ }^{\text {a }}$ \\ Habiba Bougherara PhD ${ }^{\mathrm{c}}$, Allan E. Gross MD, FRCSc ${ }^{\mathrm{b}}$, Mohammad T. Ghazavi MD, FRCSc ${ }^{\mathrm{a}}$ \\ a Department of Orthopedic Surgery, Shafa Hospital, Tehran University of Medical Sciences, Tehran, Iran \\ b Division of Orthopedic Surgery, Mount Sinai Hospital, University of Toronto, Toronto, ON, Canada \\ c Department of Mechanical and Industrial Engineering, Ryerson University, Toronto, ON, Canada
}

\section{A R T I C L E I N F O}

\section{Article history:}

Received 7 March 2012

Accepted 20 July 2012

\section{Keywords:}

hip center of rotation

dysplasia

joint reaction force

abductor muscle force

\begin{abstract}
A B S T R A C T
To study the direction and biomechanical consequences of hip center of rotation (HCOR) migration in Crowe type III and VI hips after total hip arthroplasty, post-operative radiographs and CT scans of several unilaterally affected hips were evaluated. Using a three-dimensional model of the human hip, the HCOR was moved in all directions, and joint reaction force (JRF) and abductor muscle force (AMF) were calculated for single-leg stance configuration. Comparing to the normal side, HCOR had displaced medially and inferiorly by an average of $23.4 \%$ and $20.8 \%$, respectively, of the normal femoral head diameter. Significant decreases in JRF (13\%) and AMF (46.13\%) were observed in a presumptive case with that amount of displacement. Isolated inferior displacement had a small, increasing effect on these forces. In Crowe type III and IV hips, the HCOR migrates inferiorly and medially after THA, resulting in a decrease in JRF, AMF, and abductor muscle contraction force.
\end{abstract} (c) 2013 Elsevier Inc. All rights reserved.
The location of the hip center of rotation (HCOR) following total hip arthroplasty (THA) is an essential factor that could determine the hip joint reaction force (JRF) and the abductor muscle force (AMF) by changing the moment arm of the abductor muscles [1,2]. This, in turn, predicts the wear rate and loosening risk of a hip arthroplasty as well as the amount of energy consumed by the abductor muscles during gait [3]. Most studies concur that the true acetabulum is the optimal position for the cup and results in the best outcome [4-6]. However, from a practical point of view, an anatomical positioning of the cup during THA is sometimes difficult to achieve due to acetabular dysplasia and bone deficiency [6,7]. In this regard, investigating the effect of different cup positions could help surgeons improve the outcome of the surgeries. Extensive studies have been performed on superior and lateral displacement of the HCOR, mostly after arthroplasties on Crowe type I and II dysplastic hips [8]. Muller [9], Charnley and Feagin [10], and Gore et al. [11] suggested medial transferring of the cup to decrease JRF. Russotti and Harris [5], Schutzer and Harris [12], and Tanzer [13] suggested superior placement of the cup as an alternative when placement in the anatomical position is not feasible. On the other hand, some studies

The Conflict of Interest statement associated with this article can be found at http:// dx.doi.org/10.1016/j.arth.2012.07.042.

Reprint requests: Saeid Samiezadeh, PhD Candidate, Department of Mechanical and Industrial Engineering, Ryerson University, 350 Victoria Street, Toronto, Ontario, Canada M5B 2K3. yielded poor results when placing the cup in a superior position [3,11,14-16]. Johnston et al. [17] developed a mathematical model of the hip joint to evaluate the effects of surgically achievable mechanical variables such as cup position. They found a $20 \%$ increase in JRF and $116 \%$ increase in AMF during the normal gait with $2 \mathrm{~cm}$ lateral, $2 \mathrm{~cm}$ superior, and $1 \mathrm{~cm}$ posterior displacement of the acetabular cup. Asayama et al. [18,19] and Kiyama et al. [20] suggested a superior and medical cup positioning by measuring abductor muscle strength. Delp et al. [6], using a computer simulation, showed that supero-lateral displacement of the cup adversely affected the abductor muscles by decreasing their moment arm. Lecerf et al. [21] proposed a series of clinical, radiological, and anatomical concepts and techniques regarding the femoral offset and the measurement process.

A number of studies have investigated the supero-lateral position of the cup using biomechanical models [7,20,22,23]. Nevertheless, we are not aware of any quantitative studies on the amount and direction of HCOR displacement during arthroplasty of a hip with high-riding dislocation (i.e. Crowe type III or IV) [8]. Moreover, there are few data on the biomechanical impact of the inferior transfer of the cup. As yet, there have been no studies to date that have investigated differences in abductor muscle length and preload changes with varying HCOR positions. The present study was designed to quantitatively investigate the amount of HCOR displacement in different anatomical directions during arthroplasty of dislocated joints, and the biomechanical consequences considering the effect of abductor muscle length on the muscle tension. 


\section{Materials and Methods}

This study consisted of two main parts, radiological and biomechanical. The radiological study aimed at finding the average position of HCOR after placing the cup in the true acetabulum in a congenitally dislocated hip. We evaluated the pelvic x-rays of 12 cases with unilateral Crowe III or IV hip dislocation and contra-lateral normal hip that had undergone THA on the dislocated side. We included only those cases with no history of surgery or trauma before THA with the acetabular cup placed at the true acetabulum during THA. Cases with non-standard x-rays and those with deformed pelvises were not included. The radiographs were studied regarding the amount of HCOR displacement compared to the normal side in two planes (medial-lateral and superior-inferior). We used a modification of a standard method considering the inter-ischial line as the reference for superior-inferior displacement and the vertical bisector of the pelvis for medial-lateral displacement [24]. The displacements were expressed as the percentage of the normal femoral head diameter to nullify the confounding effects of patient body size. Fig. 1 provides a radiological example illustrating this method of measurement for HCOR displacement. The medial-lateral and superior-inferior displacements were measured using the following equations. The abbreviations are explained in the figure legend.

Medial-Lateral Displacement $(\%)=\frac{L_{2}-L_{1}}{D_{2}} \times 100$

Superior-Inferior Displacement $(\%)=\frac{Y}{D_{2}} \times 100$

Post-operative pelvic CT scans of three cases were also available, and these were used to measure the anteroposterior displacement of the HCOR while comparing with the normal side using Mimics software (Materialise, Leuven, Belgium) on the digital files. We then calculated the average amount of HCOR displacement in the three aforementioned directions.

In the second part of the study, the biomechanical consequences of the displacement of the HCOR, such as changes in JRF and AMF, were calculated. Three-dimensional (3D) models of the human hip, with the gluteus medius muscle as the main abductor muscle, were developed in SolidWorks software (Dassault Systèmes SolidWorks Corp, MA) based on a previously published model [25]. In this model, the gluteus medius muscle is created with three straight lines connecting the insertion point of the femur to three different points

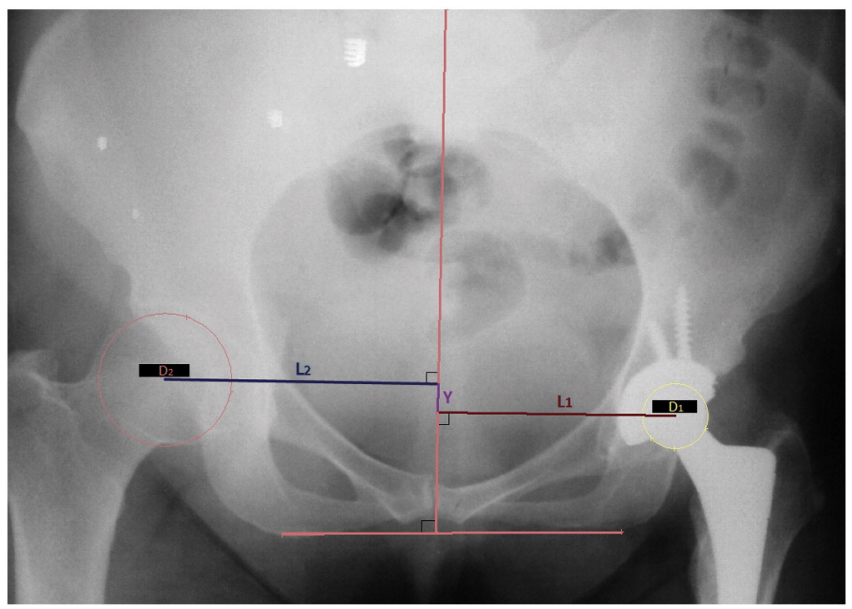

Fig. 1. Calculating the HCOR displacement following arthroplasty. The parameters used to measure the displacements are illustrated. $D_{2}$ represents the diameter of the normal femoral head. $L_{1}$ and $L_{2}$ are the distances of the two head centers to the bisector of the pelvis. on the ilium due to the large area of the origin of this muscle (Fig. 2). This assumption enhanced the accuracy of the results by forming a more realistic model of the origin area.

The HCOR was moved in the three aforementioned directions in 5$\mathrm{mm}$ increments, and JRF and AMF were calculated for single-leg stance configuration by solving the equilibrium equations using a code developed in MATLAB (The Mathworks Inc.). Biomechanical constraints were applied to the model to match it with the actual anatomy following displacements. The typical position of the HCOR following THA of a dislocated hip was then assessed for its biomechanical characteristics.

AMF was defined as the total force exerted by the abductor muscle in order to neutralize the effect of weight and satisfy equilibrium conditions. It included the muscle preload and the muscle contraction force $(\mathrm{CF})$. The preload increases once the muscle is placed under more tension, and decreases when the muscle length decreases. The CF was defined as the force the muscle produces so that the total force would be equal to AMF. To assess the potential effect of HCOR displacement on muscle tension and preload, the changes in the length of the three abductor muscle bundles were calculated for different positions of HCOR in superior-inferior, medial-lateral and anterior-posterior directions.

\section{Results}

Studying 640 possible HCOR locations (based on combinations of three-planar displacement) showed that HCOR altered muscle forces and JRF significantly. To account for variability in patient weight, we calculated the JRF and AMF of different positions in the scale of the normal side measures. Our results demonstrated that the displacement would cause the JRF and AMF to change by up to $40 \%$ and $150 \%$, respectively. Specifically, medial displacement of the HCOR decreases JRF and AMF significantly, and moving it distally increases them to a slight degree. Interestingly, moving in the anterior-posterior direction does not have a significant effect on the forces in single-leg stance configuration. Figs. 3 and 4 represent the details including any single combination of the HCOR displacement and the resultant change in JRF and AMF. The results showed that the more medial the HCOR, the less the changes in the JRF and AMF would be with moving the cup in

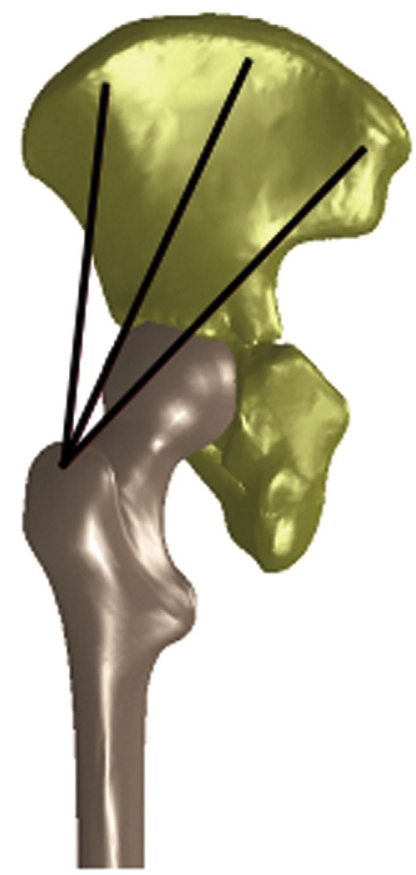

Fig. 2. The 3-D model used to represent the femur and the abductor muscle. 

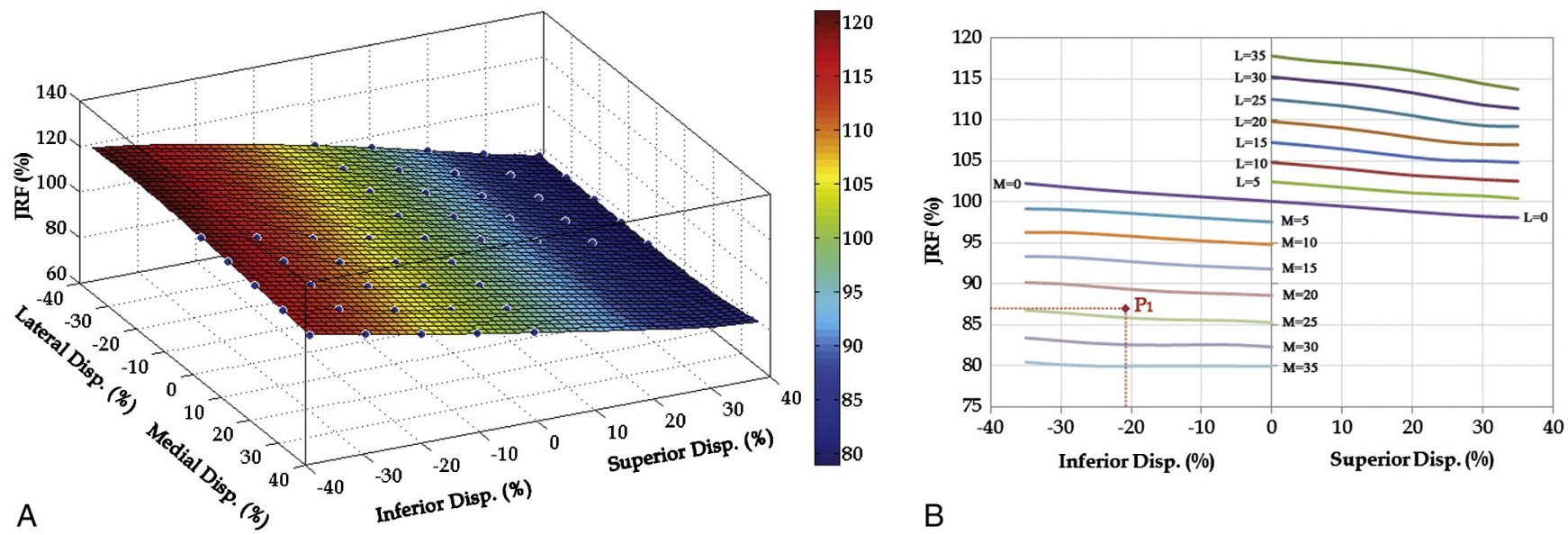

B

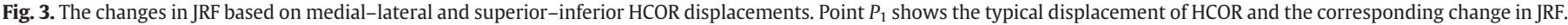

superior-inferior direction. For example, as depicted in Fig. 3, at 35\% lateral displacement, the maximum change in the forces with superior-inferior direction is $12.3 \%$. However, at $35 \%$ medial displacement, this change is very small (2.8\%).

In each location, the change in muscle length compared to the neutral position of the cup was calculated to investigate the effect of preload on CF. Medial displacement decreased the abductor muscle average length, while inferior displacement increased its length, but not equally. For instance, in neutral anterior-posterior position, a pure $20 \%$ inferior displacement would cause an average of $6.3 \%$ increase in abductor muscle length, whereas moving $20 \%$ medially would cause an average of $2.7 \%$ decrease in the abductor muscle length. Combining the two, a 20\% inferior and medial displacement would cause an average of $3.7 \%$ increase in muscle length. Anterior-posterior displacement of HCOR can result in controversial changes in the muscle length depending on the size of displacement in the other two directions, as depicted in Table 1. This table represents a number of combinations and the resulting abductor muscle stretch. The muscle stretch was represented as a percentage of the muscle length when the cup is in the normal anatomic position.

The measurements on radiographs showed a typical medial displacement by an average of $23.4 \%$ of femoral head diameter $(D)$ and an inferior displacement by an average of $20.8 \%$ of $D$ following arthroplasty. Also, up to $9.6 \% \mathrm{D}$ (average 6.4\%) anterior displacement was detected in CT scan measurements.
This typical displacement caused $13 \%$ and $46.13 \%$ reduction in JRF and AMF, respectively, when compared with a normal HCOR. Furthermore, an average of 3.3\% increase in abductor muscle length was calculated for this typical displacement.

Isolated calculations on the effect of unidirectional transfer of the HCOR were performed as well, revealing $13.6 \%$ and $48.9 \%$ reduction in JRF and AMF, respectively, with $23.4 \%$ medialization without any distalization. Conversely, a $1.2 \%$ and $3.8 \%$ increase in JRF and AMF was observed for $20.8 \%$ distalization without any medialization. Pure anterior displacement of the HCOR did not show any impact on the forces. More comprehensive data including the changes of JRF and AMF for every possible combination of mediallateral and superior-inferior displacement of HCOR are presented in Figs. 3 and 4, respectively.

\section{Discussion}

Total hip arthroplasty is one of the most successful reconstructive surgeries and can greatly improve a patient's quality of life. The success of this surgery in treating neglected developmentally dislocated hips has been well established for some time [10], but the function of these patients, as well as the survival of the prostheses, has not been shown to be as satisfactory as in simple osteoarthritis $[4,26]$. This is partly due to the bone deficiency of the hip, especially the acetabular side that makes the artificial joint more susceptible to loosening $[27,28]$. Also, the patients with hip subluxation or
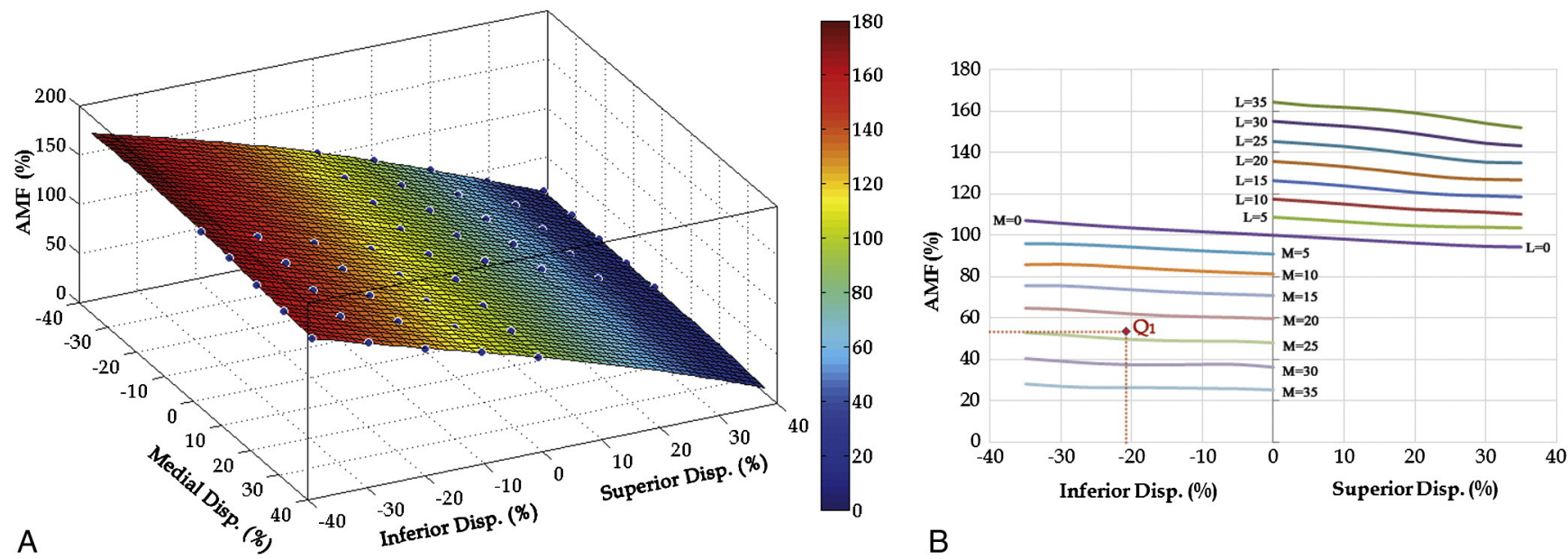

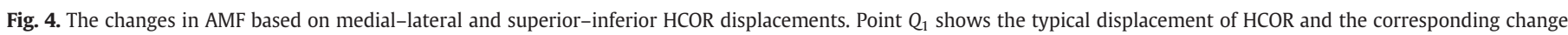
in AMF. 
Table 1

Abductor muscle stretch as a function of some common combinations of HCOR displacement in three anatomic directions.

\begin{tabular}{|c|c|c|c|c|c|c|}
\hline $\begin{array}{l}\text { S-I* } \\
\text { Disp. } \\
(\%)\end{array}$ & $\begin{array}{l}\text { M-L* } \\
\text { Disp. } \\
(\%)\end{array}$ & $\begin{array}{l}\text { A-P* } \\
\text { Disp. } \\
(\%)\end{array}$ & $\begin{array}{l}\text { Muscle } \\
\text { Stretch } \\
\text { (Anterior) } \\
(\%)\end{array}$ & $\begin{array}{l}\text { Muscle } \\
\text { Stretch } \\
\text { (Middle) } \\
(\%)\end{array}$ & $\begin{array}{l}\text { Muscle } \\
\text { Stretch } \\
\text { (Posterior) } \\
(\%)\end{array}$ & $\begin{array}{l}\text { Average } \\
\text { Stretch } \\
(\%)\end{array}$ \\
\hline 0 & -30 & 0 & 3.1 & 4.4 & 6.6 & 4.7 \\
\hline 10 & -30 & 0 & -0.5 & 1.6 & 3.9 & 1.6 \\
\hline 30 & -30 & 0 & -7.5 & -4.1 & -1.6 & -4.4 \\
\hline 0 & -10 & 0 & 0.9 & 1.4 & 2.2 & 1.5 \\
\hline 10 & -10 & 0 & -2.7 & -1.5 & -0.7 & -1.6 \\
\hline 30 & -10 & 0 & -9.8 & -7.2 & -6.3 & -7.8 \\
\hline-30 & 0 & 0 & 10.9 & 8.8 & 8.9 & 9.5 \\
\hline-10 & 0 & 0 & 3.6 & 2.9 & 2.9 & 3.2 \\
\hline 0 & 0 & 0 & 0.0 & 0.0 & 0.0 & 0.0 \\
\hline-30 & 10 & 0 & 10.1 & 7.5 & 7.0 & 8.2 \\
\hline-10 & 10 & 0 & 2.8 & 1.6 & 0.9 & 1.8 \\
\hline 0 & 10 & 0 & -0.8 & -1.4 & -2.1 & -1.4 \\
\hline-30 & 30 & 0 & 8.9 & 5.2 & 3.2 & 5.7 \\
\hline-10 & 30 & 0 & 1.6 & -0.9 & -3.0 & -0.8 \\
\hline 0 & 30 & 0 & -2.0 & -3.9 & -6.1 & -4.0 \\
\hline-20.8 & 23.4 & 6.4 & 5.1 & 2.9 & 2.0 & 3.3 \\
\hline-30 & 0 & 10 & 9.7 & 8.5 & 9.6 & 9.3 \\
\hline-10 & 0 & 10 & 2.3 & 2.6 & 3.6 & 2.9 \\
\hline 0 & 0 & 10 & -1.3 & -0.3 & 0.7 & -0.3 \\
\hline-30 & 10 & 10 & 8.9 & 7.2 & 7.6 & 7.9 \\
\hline-10 & 10 & 10 & 1.5 & 1.3 & 1.6 & 1.5 \\
\hline 0 & 10 & 10 & -2.1 & -1.7 & -1.4 & -1.7 \\
\hline-30 & 30 & 10 & 7.7 & 4.8 & 3.8 & 5.4 \\
\hline-10 & 30 & 10 & 0.3 & -1.2 & -2.3 & -1.1 \\
\hline 0 & 30 & 10 & -3.3 & -4.2 & -5.4 & -4.3 \\
\hline 0 & -30 & 0 & 3.1 & 4.4 & 6.6 & 4.7 \\
\hline 10 & -30 & 0 & -0.5 & 1.6 & 3.9 & 1.6 \\
\hline
\end{tabular}

Values in italic depict the measurements for the typical position of HCOR.

* S-I (superior-inferior), M-L (medial-lateral), A-P (anterior-posterior). Superior, medial, and anterior directions are defined as positive.

dislocation usually suffer from a severe limp before the surgery that may continue to a lesser severity after THA [29]. This is mostly the result of hip abductor muscle malfunction, which is due to their chronically shortened condition and subsequent atrophy $[4,27]$. Considering these facts, any technique that decreases the hip JRF and AMF following THA of the Crowe type III and IV hips could potentially improve the function, and also the survival, of the joint.

The unique effect of the HCOR on the forces imposed to the hip joint has been well documented $[7,20,23]$. Specifically, the consequences of lateral and superior displacement of HCOR that can happen during THA of a Crowe I or II have been investigated in previous studies $[5,7,12,13,20,21,30]$. Although it is generally agreed that the anatomical position of HCOR results in the best outcome, some discrepancy in the results of clinical and biomechanical studies remains. This can partially be explained by the inherent limitations of biomechanical studies, including their inability to perfectly simulate the anatomy and physiology of the human body. Another reason is the lack of enough follow-up in some clinical studies to reveal the adverse clinical consequences of non-anatomical cup positions [12].

Importantly, little is known about the possible consequences of combined medial and inferior displacement, and this is the first study to contend with this issue. Based on the results of the current study, the typical displacement of the HCOR during THA of a congenitally dislocated hip is toward medial, inferior, and anterior. This can be explained by the underdevelopment of the true acetabulum of a congenitally dislocated hip that leaves it shallow and small in diameter, so that only a small cup can be inserted into it. Due to the fact that true acetabulum is located just superior to the obturator foramen, the smaller diameter of the cup is translated into a lower HCOR. Another factor that may increase this distalization in Crowe III hips is the presence of the osteophytes sitting at the inferior margin of the false acetabulum.
At least two different reasons can be mentioned for medial displacement of the HCOR in arthroplasty of dislocated hips. First, any decrease in the diameter of the cup means that the distance between the medial wall of the acetabulum and the HCOR will also decrease. Second, the funnel-shaped geometry of the bony pelvis induces some medialization with any inferior transfer of the HCOR.

Our findings regarding anterior transfer of the HCOR are in agreement with a previous study [31]. That may be attributable to the general mal-development of the acetabulum in the absence of a reciprocal femoral head. In addition, the results regarding the net superior displacement are consistent with those obtained from previous studies [6].

We found that after arthroplasty of a typical Crowe III or IV hip, the JRF is less than the normal side by $13 \%$. It would be even more reduced (1.2\% averagely) if the inferior displacement of the HCOR could be avoided, but technical limitations usually restrict the freedom of the surgeon in this case. However, any decrease in inferior displacement of the HCOR is potentially rewarded by a longer-lasting arthroplasty. The surgeon plays an especially important role in avoiding lateralization of the HCOR, which is a possible technical error if the medial acetabular osteophytes are not addressed properly. Any lateralization of HCOR directly increases the JRF to a large extent. It also indirectly increases the JRF to a lesser degree by magnifying the effect of inferior HCOR transfer.

Another important finding was the remarkable decrease in the AMF by $46.13 \%$. Considering the weakness of the abductor muscles of dislocated hips, this amount of reduction in AMF is of paramount importance. It simply means that for stabilizing the pelvis during gait, hip abductors have to produce $46.13 \%$ less force in comparison with the normal side. This finding explains the significant improvement of the Trendelenburg lurch of the patients after THA [32]. Although some of the patients continue to have some limp after surgery [30], the limping would be much worse in the absence of this beneficial biomechanical effect.

The impact of displacing the HCOR on the abductor muscle preload should be noted. Although quantitative calculation of the preload changes was not possible due to lack of data on stiffness of this muscle, the changes in the muscle length are an indirect qualitative indicator of preload changes. Stretching the muscle increases the preload and vice versa [33]. Since during single-leg stance the AMF neutralizes body weight, it is thus a function of body weight [34]. The preload constitutes a part of the whole force that is needed to resist the body weight (i.e. AMF). Any changes in the preload will be compensated by reverse changes in the muscle contraction force $(\mathrm{CF})$ [35]. JRF is also directly calculated from AMF. Therefore, in single-leg stance position, preload changes do not affect the size of either the AMF or JRF, but rather the CF. However, whenever no AMF is necessary for function (i.e. resting position) an increase in preload could result in the creation of unnecessary JRF.

The typical position of the HCOR after placing the cup in true acetabulum results in a slight increase $(3.3 \%)$ in the muscle length (Table 1) and a corresponding increase in the preload that, as explained before, does not affect the AMF or JRF during single-leg stance, but decreases the $C F$ necessary for pelvis stabilization. This decrease in the $\mathrm{CF}$ is another favorable event regarding the patient's gait.

The present study has some important advantages. To the best of our knowledge, this is the first study on the amount and direction of HCOR displacement following arthroplasty of a Crowe III or IV hip. In addition, some biomechanical considerations have been neglected in previous studies. For example, a single point has been usually assumed as the origin of the abductors. The current study used an advanced 3-D model of the musculoskeletal system of the hip, which improved the validity of the biomechanical measurements. Taking the true area of the origin of the abductor muscles into consideration enabled us to accurately measure the resulted changes. The effect of HCOR displacement on muscle preload and CF was also investigated. 
Some limitations can also be mentioned for this study. Quantitative investigation of the $\mathrm{CF}$ changes could not be performed due to the lack of data in passive stiffness properties of the abductor muscle. However, mathematical measurements in typical displacement of HCOR showed a slight increase in muscle length and an increase in muscle preload, and, as a result, a further decrease in CF. In addition, only the gluteus medius muscle was investigated. Although it was ideal to involve other abductor muscles in the study, the calculations would be very complicated. Considering the superior role of gluteus medius as the most important abductor muscle of the hip, this simplification has not probably changed the results significantly [31]. Furthermore, the study was limited to a static analysis in single-leg stance position. Although this is the traditional position for biomechanical studies, and probably the most important one as well, the forces through the hip joint may behave differently in other positions and in dynamic analyses. For example, the seemingly innocent anterior displacement of the HCOR may not be so harmless in the loaded flexed position of the hip, like when standing up from a sitting position. Also, in contrast to the single-leg stance, changes in muscle preload can cause JRF alteration in some other positions as explained previously. Finally, it should be noted that static biomechanical studies cannot consider some physiological issues, such as muscle deconditioning or endurance, and the effect of dynamic forces and thus should not be considered as absolute predictors of the joint kinetics.

According to our results, after placing the cup in the true acetabulum of a Crowe III or IV dysplastic hip, the HCOR will typically move inferiorly, medially, and anteriorly in comparison to the normal HCOR. This displacement typically decreases the JRF, AMF and CF and slightly increases the abductor muscle preload. The inferior movement of the HCOR increases the JRF and AMF to a slight degree that is generously compensated by the beneficial impact of the simultaneous medial transfer which reduces the JRF and AMF significantly. The single most crucial event to avoid is lateralization of the HCOR, which increases the JRF and AMF directly, as well as indirectly, by potentiating the adverse effect of the inferior displacement. Anterior displacement has no measurable effect on AMF and JRF and very little influence on the muscle preload and CF. The combined inferior and medial displacement of the HCOR, which is typical during THA of a congenitally dislocated hip, is helpful biomechanically. However, if the inferior displacement can be avoided, a slightly more decrease in AMF and JRF will be anticipated.

\section{Acknowledgments}

We wish to thank Mr. Richard Barbuto, Mrs. Akbari, and Mr. Mahdi Hematabadi for their kind contribution to this study. The financial support received from Ryerson University as "Health Research Fund" is acknowledged.

\section{References}

1. Cereatti A, Donati M, Camomilla V, et al. Hip joint centre location: an ex vivo study. J Biomech 2009;42:818.

2. Erceg M. The influence of femoral head shift on hip biomechanics: additional parameters accounted. Int Orthop 2009;33:95.

3. Pagnano W, Hanssen AD, Lewallen DG, et al. The effect of superior placement of the acetabular component on the rate of loosening after total hip arthroplasty. J Bone Joint Surg Am 1996;78:1004.

4. Linde F, Jensen J. Socket loosening in arthroplasty for congenital dislocation of the hip. Acta Orthop Scand 1988;59:254.
5. Russotti GM, Harris WH. Proximal placement of the acetabular component in total hip arthroplasty. A long-term follow-up study. J Bone Joint Surg Am 1991; 73:587.

6. Delp SL, Wixson RL, Komattu AV, et al. How superior placement of the joint center in hip arthroplasty affects the abductor muscles. Clin Orthop Relat Res 1996:137.

7. Doehring TC, Rubash HE, Shelley FJ, et al. Effect of superior and superolateral relocations of the hip center on hip joint forces. An experimental and analytical analysis. J Arthroplasty 1996;11:693.

8. Crowe JF, Mani VJ, Ranawat CS. Total hip replacement in congenital dislocation and dysplasia of the hip. J Bone Joint Surg Am 1979;61:15.

9. Muller ME. Total hip prostheses. Clin Orthop Relat Res 1970;72:46.

10. Charnley J, Feagin JA. Low-friction arthroplasty in congenital subluxation of the hip. Clin Orthop Relat Res 1973:98.

11. Gore DR, Murray MP, Gardner GM, et al. Roentgenographic measurements after Muller total hip replacement. Correlations among roentgenographic measurements and hip strength and mobility. J Bone Joint Surg Am 1977;59:948.

12. Schutzer SF, Harris WH. High placement of porous-coated acetabular components in complex total hip arthroplasty. J Arthroplasty 1994;9:359.

13. Tanzer M. Role and results of the high hip center. Orthop Clin North Am 1998;29: 241.

14. Callaghan JJ, Salvati EA, Pellicci PM, et al. Results of revision for mechanical failure after cemented total hip replacement, 1979 to 1982. A two to five-year follow-up. J Bone Joint Surg Am 1985;67:1074.

15. Kelley SS. High hip center in revision arthroplasty. J Arthroplasty 1994;9:503.

16. Morag G, Zalzal P, Liberman B, et al. Outcome of revision hip arthroplasty in patients with a previous total hip replacement for developmental dysplasia of the hip. J Bone Joint Surg Br 2005;87:1068.

17. Johnston RC, Brand RA, Crowninshield RD. Reconstruction of the hip. A mathematical approach to determine optimum geometric relationships. J Bone Joint Surg Am 1979;61:639.

18. Asayama I, Chamnongkich S, Simpson KJ, et al. Reconstructed hip joint position and abductor muscle strength after total hip arthroplasty. J Arthroplasty 2005;20: 414.

19. Asayama I, Naito M, Fujisawa M, et al. Relationship between radiographic measurements of reconstructed hip joint position and the Trendelenburg sign. J Arthroplasty 2002;17:747.

20. Kiyama T, Naito M, Shitama H, et al. Effect of superior placement of the hip center on abductor muscle strength in total hip arthroplasty. J Arthroplasty 2009;24:240.

21. Lecerf G, Fessy MH, Philippot R, et al. Femoral offset: anatomical concept, definition, assessment, implications for preoperative templating and hip arthroplasty. Orthop Traumatol Surg Res 2009;95:210.

22. Jerosch J, Steinbeck J, Stechmann J, et al. Influence of a high hip center on abductor muscle function. Arch Orthop Trauma Surg 1997;116:385.

23. Lengsfeld $M$, Bassaly A, Boudriot $U$, et al. Size and direction of hip joint forces associated with various positions of the acetabulum. J Arthroplasty 2000;15: 314.

24. Kirkwood RN, Culham EG, Costigan P. Radiographic and non-invasive determination of the hip joint center location: effect on hip joint moments. Clin Biomech (Bristol, Avon) 1999;14:227.

25. Dostal WF, Andrews JG. A three-dimensional biomechanical model of hip musculature. J Biomech 1981;14:803.

26. Sanchez-Sotelo J, Berry DJ, Trousdale RT, et al. Surgical treatment of developmental dysplasia of the hip in adults: II. Arthroplasty options. J Am Acad Orthop Surg 2002:10:334.

27. Inao S, Matsuno T. Cemented total hip arthroplasty with autogenous acetabular bone grafting for hips with developmental dysplasia in adults: the results at a minimum of ten years. J Bone Joint Surg $\mathrm{Br}$ 2000;82:375.

28. Saleh KJ, Jaroszynski G, Woodgate I, et al. Revision total hip arthroplasty with the use of structural acetabular allograft and reconstruction ring. J Arthroplasty 2000;15:951.

29. Stans AA, Pagnano MW, Shaughnessy WJ, et al. Results of total hip arthroplasty for Crowe type III developmental hip dysplasia. Clin Orthop Relat Res 1998:149.

30. Kanai A, Kiyama T, Genda E, et al. Biomechanical investigation of ambulatory training in patients with acetabular dysplasia. Gait Posture 2008;28:52.

31. Beck M, Sledge JB, Gautier E, et al. The anatomy and function of the gluteus minimus muscle. J Bone Joint Surg Br 2000;82:358.

32. Eskelinen A, Helenius I, Remes V, et al. Cementless total hip arthroplasty in patients with high congenital hip dislocation. J Bone Joint Surg Am 2006;88:80.

33. Garrett Jr WE, Safran MR, Seaber AV, et al. Biomechanical comparison of stimulated and nonstimulated skeletal muscle pulled to failure. Am J Sports Med 1987; $15: 448$

34. Papavasiliou VA. Lateral and distal transfer of the greater trochanter. Preliminary observations on a conservative surgical treatment for the osteoarthritic hip. Clin Orthop Relat Res 1986:198.

35. Rhoades RA BD. Medical physiology: principles for clinical medicine. 4th ed. Lippincott Williams \& Wilkins; 2012. 